\title{
THE EFFECT OF NANOVOID ELONGATION ON ELECTRON MULTIPLICATION DURING (SUB)NANOSECOND ELECTRICAL BREAKDOWN
}

\author{
1'Ján TUNGLI, ${ }^{1}$ Petr BÍLEK, 'deněk BONAVENTURA \\ ${ }^{1}$ Department of Physical Electronics, Fac. Sci., Masaryk University, Brno, Czech Republic, EU, \\ tun@mail.muni.cz
}

https://doi.org/10.37904/nanocon.2020.3726

\begin{abstract}
We investigate how the geometry of nanovoids present during the (sub)nanosecond electrical breakdown in liquid water influences the electron multiplication. Our analysis uses a particle-tracing Monte Carlo simulation toolkit Geant4-DNA. The electron multiplication in nanovoids is one of the possible scenarios describing the charge generation in liquid water. In our simplified model, we assume a constant homogeneous electric field, an ellipsoidal shape of nanovoids, and isotropic distribution of the primary electron's velocity. The quantity of interest is the number of ionizations per number of launched primary electrons. We study this yield of electrons as we vary the ellipsoid eccentricity parameter while keeping the volume constant. The nanovoids with zero eccentricity (spherical case) produce smaller yield since the suppressed length does not favor electron multiplication. On the other hand, a very prolonged ellipsoid causes the loss of most electrons because of early collisions with the void-liquid interface. Therefore, for a particular value of eccentricity, the yield is maximal. The results of this study contribute to the elucidation of the initial phases of discharge evolution in polar liquids and is one of the first steps towards quantitative assessment of the importance of microscopic parameters involved. The understanding of this phenomenon promises the explanation of macroscopic parameters observed in the (sub)nanosecond electrical breakdown experiments
\end{abstract}

Keywords: Nanovoids, electrostriction, liquid water electric breakdown, nanosecond pulsed electric field

\section{INTRODUCTION}

Historically, plasma discharges has been used as a medium to generate chemically active species in various gas mixtures. During past few decades, attempts to generate plasma also in liquid environment has emerged, followed immediately by applications such as nanomaterials syntheses. Research of pulsed discharges in water is of interest to many applications. These include: purification of hazardous organic compounds [1,2], high power switch systems [3], and electric impulse fragmentation applied in recycling [4], or electrohydraulic lithotripsy [5].

Dielectric fluids in inhomogeneous electric field are subject to ponderomotive forces [6]. In a homogeneous fluid and in the absence of free electric charge this force causes the fluid to move to regions with higher electric field. However, if the electric pulse is strong and fast enough, the fluid does not have time to move and large negative pressures appear that can lead to cavitation [7]. It is known that with application of a strong nanosecond electric pulse, discharge develops. During these small time scales a model where the water is heated up and the discharge is created in a resultant gas bubble can be shown to be inconsistent with experimental findings [8]. Explaining the initial stages of discharge formation therefore involves processes occurring at sub-nanosecond and sub-micrometer scales and makes the topic challenging.

One of the models worth investigating is the electron avalanche formation inside nanovoids formed by the cavitation process. Electrons ejected into a nanovoid could gain enough energy to ionize water molecules upon collision. Although the shape and size of these nanovoids is still unknown, cavitation models usually 
assume a spherical shape. Furthermore, in the presence of electric field, a spherical void would become elongated in the direction of electric field and so to assume an ellipsoidal shape is reasonable. In this work, we study electron multiplication occurring inside an ellipsoidal nanovoid by means of particle tracking Monte Carlo simulation. We focus mainly on the effect of nanovoid elongation on the average yield, which we define as the number of secondary electrons produced per the number of primary electron injected into the nanovoid.

\section{MODEL}

To accurately simulate electron acceleration and multiplication a simulation toolkit called Geant4-DNA $[9,10]$ $[11,12]$. Geant4 is an open-source project that allows simulation of particles passing through matter. The extension - Geant4-DNA - is aimed primarily at biological application and includes physical processes for liquid water. The toolkit is widely used in many fields of physics, including nuclear, space and medical research. In our setup, an ellipsoid of variable eccentricity is placed at the center of the simulation box using material Watersteam, which simulates a nanovoid that could be born during the application of highly inhomogeneous electric field. The rest of the box is filled with water, see Figure 1.

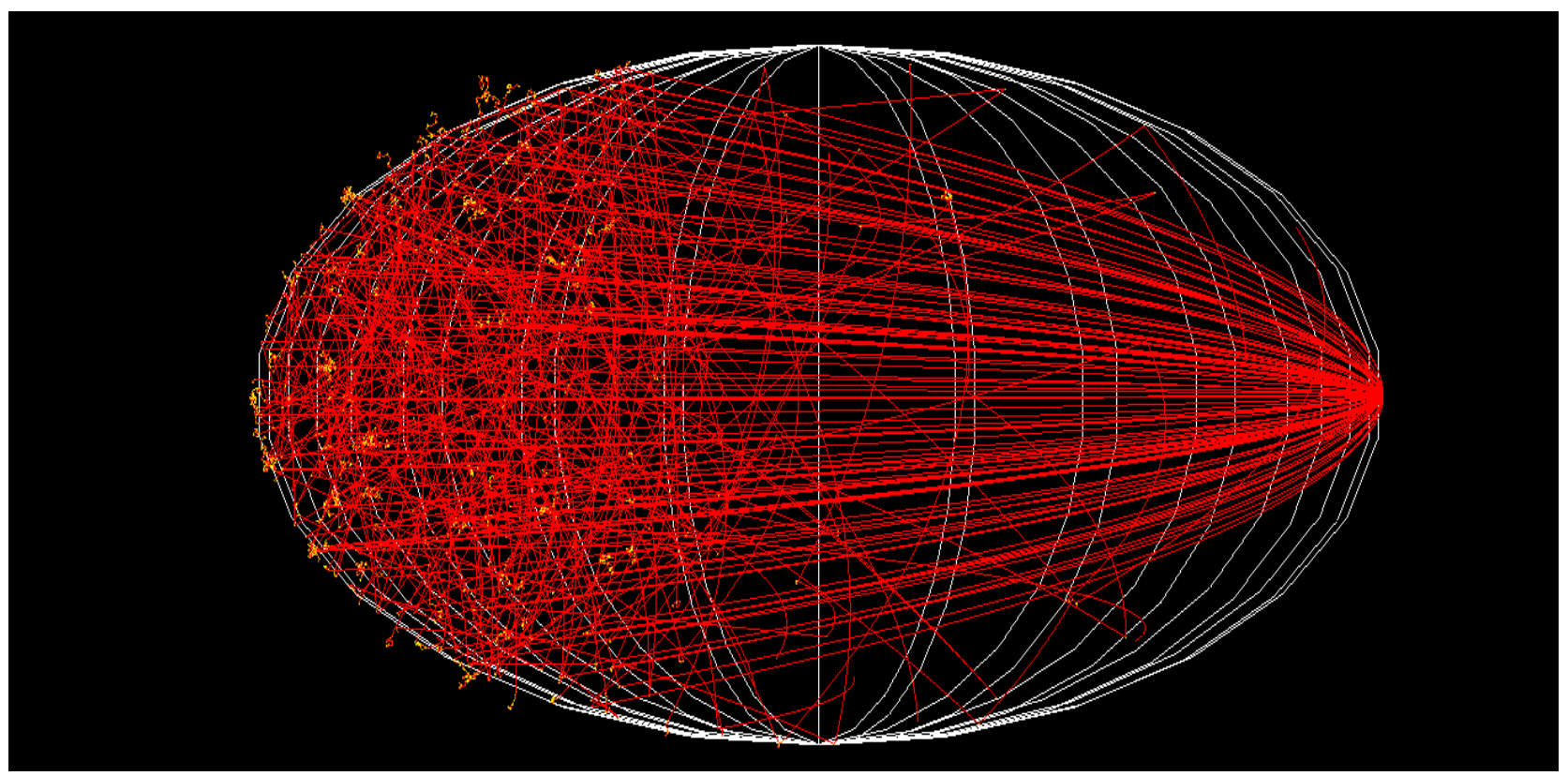

Figure $1 \mathrm{~A}$ beam of 100 electrons accelerated in an ellipsoidal void in Geant4. The trajectory of the electrons is represented with red lines while the yellow dots represent collisional events

The main purpose of this work is to study the effect of elongation of the nanovoid. In order to isolate this geometric effect on electron multiplication, the following two-step procedure is used:

1) In a sphere (ellipsoid of zero eccentricity), find the radius for which, on average, the number of electrons created is equal to the number of primary electrons, i.e, for which the yield is equal to 1 .

2) Vary the elongation of the nanovoid by changing the eccentricity in a way that the volume of the ellipsoid stays constant and monitor the yield. Also, in both cases, a constant electric field of $0.2 \mathrm{~V} / \mathrm{nm}$ is applied in the direction the ellipsoid is elongationed.

The first step is accomplished by running a series of runs with variable radius of the sphere and finding the radius $R$ where the function:

$$
f(R)=Y(R)-1
$$


is equal to zero. Each run consists of launching 10000 electrons from the ellipsoid vertex. The initial momenta of electrons are distributed isotropically and the initial energy of electrons is left as a parameter and multiple values are tested.

Once the radius is found, the second step can be undertaken. As the eccentricity is changed, the volumepreserving transformations for the ellipsoid parameters are:

$$
\begin{gathered}
a=\frac{R_{0}}{\sqrt[3]{1-e^{2}}} \\
b=\sqrt{\left(1-e^{2}\right)} a,
\end{gathered}
$$

where $a$ and $b$ are the semi-axes and $b<a$. Because of the symmetry of the problem, the third semi-axis is simply set equal to the second semi-axis (the smaller one).

In Geant4, a cut-off energy threshold for electrons propagating in bulk water needs to be specified. It is assumed that electrons in water below this energy threshold are immediately solvated. The assumptions made and their effect on the result will be discussed further below.

\section{RESULTS}

The radius where the yield is balanced is found to be approximately $0.09 \mu \mathrm{m}$ at energies about $1 \mathrm{eV}$ and decreases for higher energies. Also, as is shown in Figure 2, the yield in a sphere is approximately a linear function of radius in the range of values investigated. As the eccentricity of the ellipsoid is increased, the yield increases until it reaches a maximum value (Figure 3). Further increasing eccentricity, while maintaining constant volume, means that more electrons will not have enough time to gain energy from electric field. As expected, the lower the energy of the electron, the more it benefits from elongation since the average electron motion is more directed by the applied electric field. As a consequence, the eccentricity at which the yield is maximal is dependent on the initial energy (Figure 4).

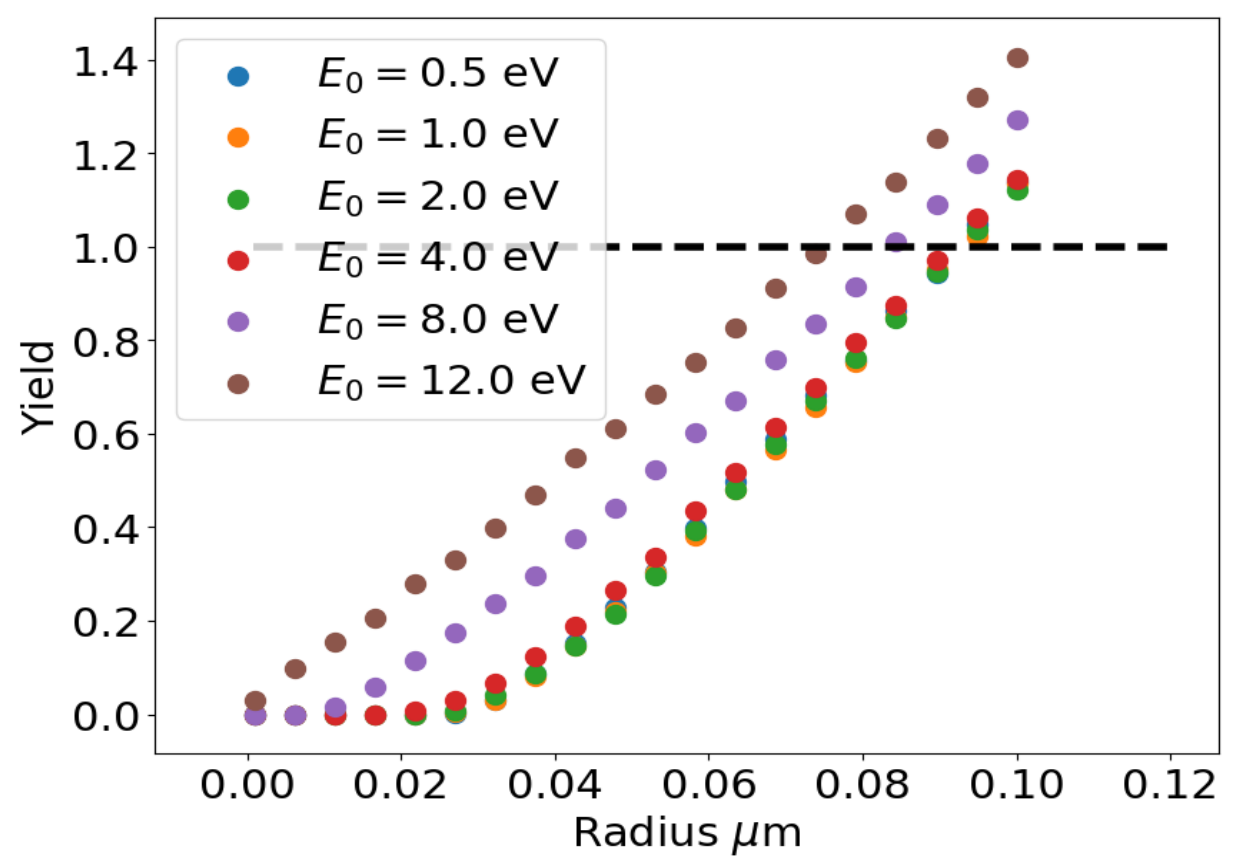

Figure 2 Secondary electron yield as a function of spherical radius 


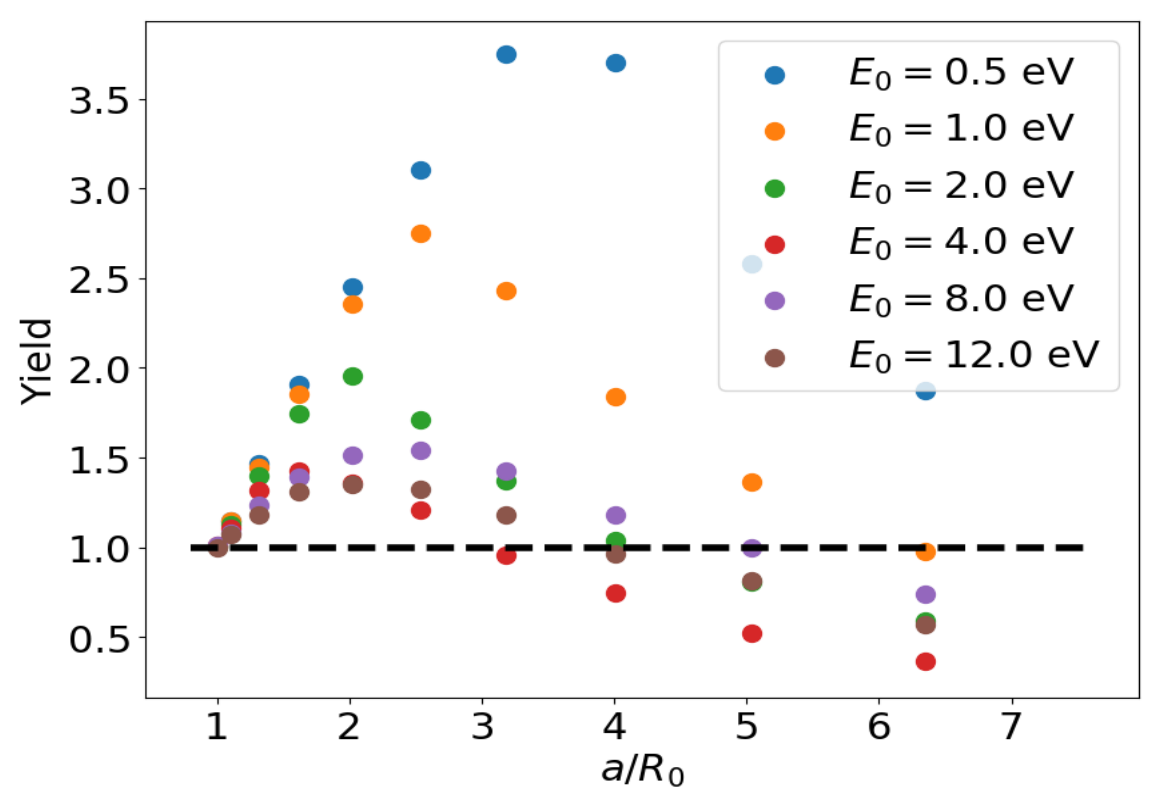

Figure 3 Secondary electron yield as a function of ellipsoid elongation

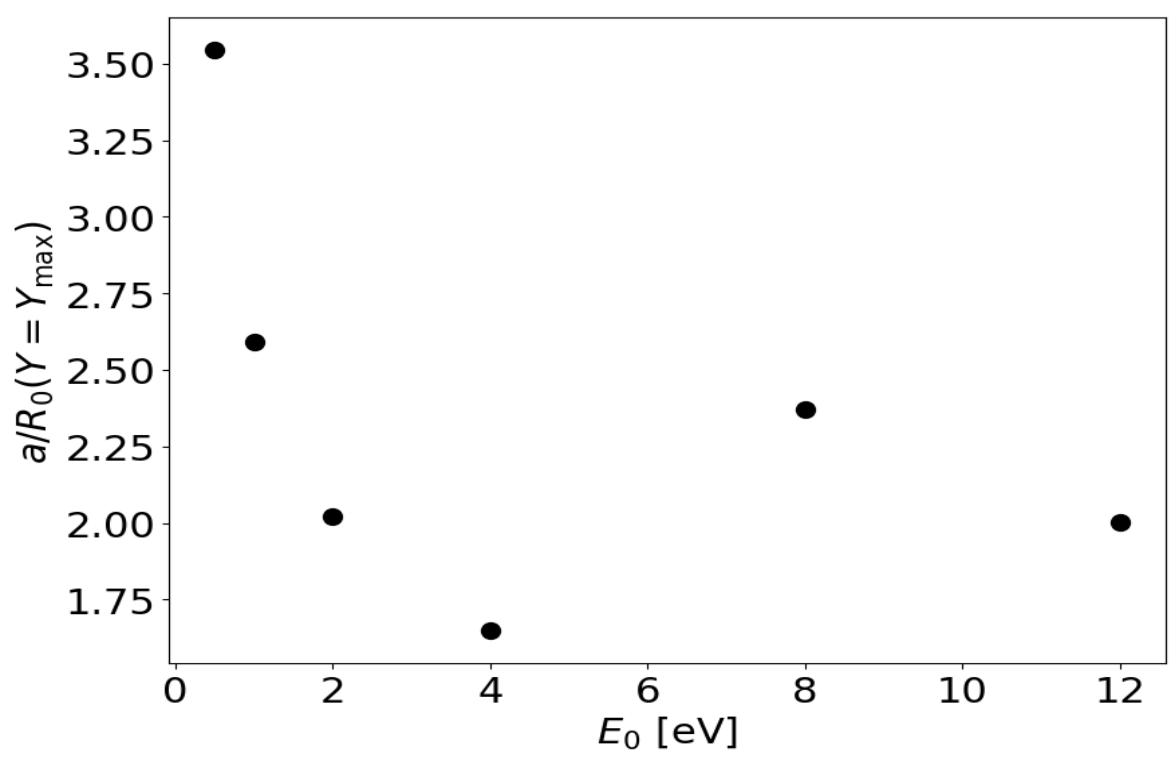

Figure 4 Elongation parameter where the yield is maximal as a function of initial energy

\section{DISCUSSION}

For a correct interpretation of the results, it is important to discuss the effects of assumed parameters in our simulations.

\section{Electron solvation}

Because of the lack of cross-sectional data for processes in water, Geant4 terminates the flight of an electron in bulk water below a certain energy threshold. The electron is assumed to be solvated. Since this energy, in our case, is well below the ionization energy, it may seem that this parameter is of no consequence on the results of the simulation. Unfortunately, this is not the case and electron solvation energy acts as an important 
numerical parameter. The effect of this parameter has been studied already by other scientists and it seems to provide quantitative correlation with experimental data the value would have to be set below the smallest value allowed by Geant4 [13]. It is therefore important to bare in mind that the secondary electron yield obtained from these simulations is only a lower limit and experimentally measure yields will most likely be larger.

\section{Initial energy and momentum distribution}

In this study, the assumption of the isotropic distribution of electron momenta is chosen for simplicity. Nonetheless, the question whether this assumption is justified remains. To our knowledge, there are no experimental data describing the properties of primary electrons originating in bulk water. Therefore, to what extend are the primary electrons guided to the void by the external electric field is unknown.

In addition, because of cavitation effects produced by inhomogeneous electric field, spatial fluctuations of electric field can play a major role in the resulting dynamics of electron propagation inside the nanovoid.

The initial energy of electrons turns out to be an important parameter. Electrons with low energy are more likely to fly longer and possibly gain more energy from the electric field. On the other hand, due to theisotropic distribution used here, electrons with higher energy can hit the bulk water surface sooner and may not have enough energy for ionization (Figure 5).

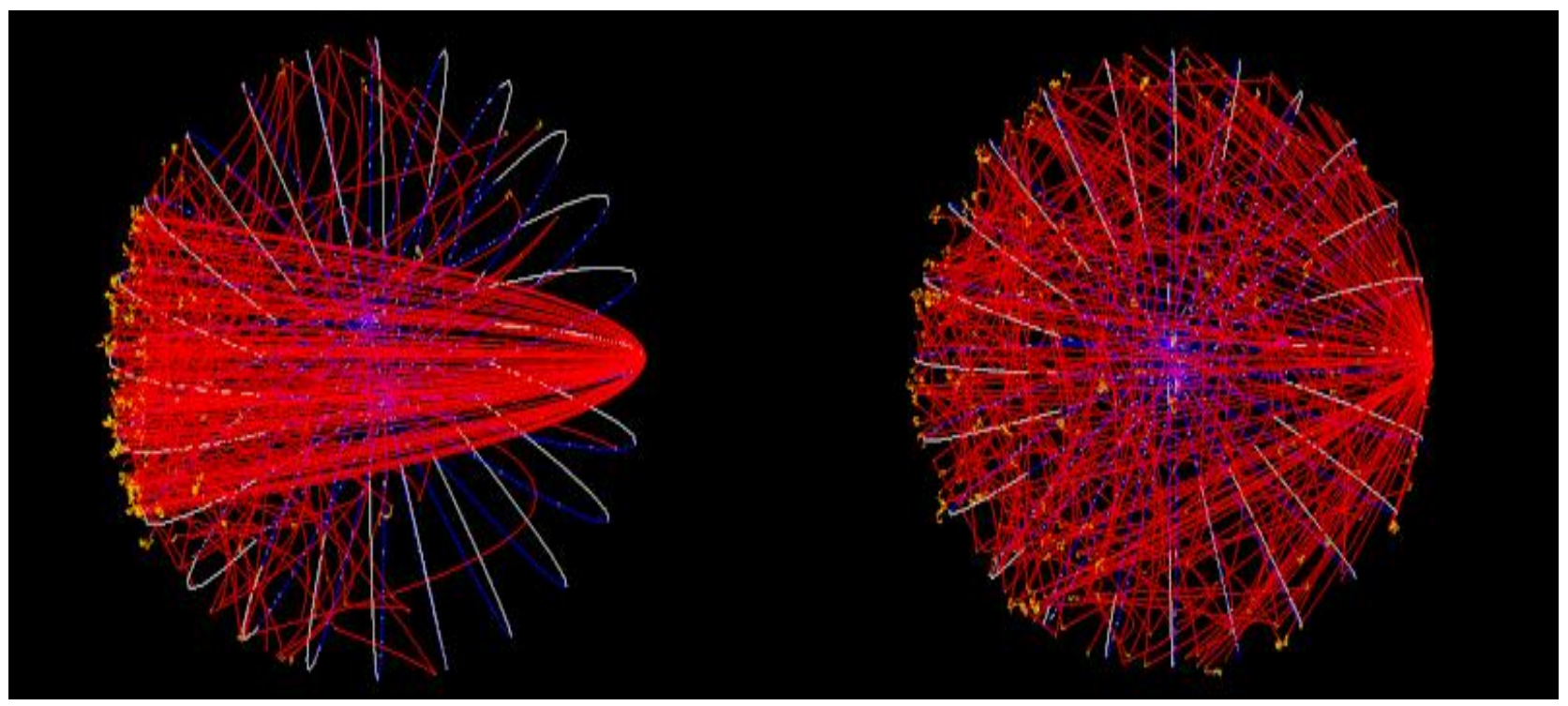

Figure 5 Electron trajectories - low initial energy (left) and high initial energy (right)

\section{CONCLUSION}

A quantitative assessment of electron multiplication inside ellipsoidal nanovoids has been done by means of particle-tracing Monte Carlo simulation. Influence of ellipsoid elongation and the effect of initial energy of electrons has been studied. From the results obtained, several conclusions are apparent:

- The initial momenta of electrons have a significant effect on the number of electrons produced.

- $\quad$ The number of secondary electrons generated in a void of ellipsoidal shape can be up to $\sim 5$ times higher compared to a spherical void of the same volume. Also, because of cavitation effects produced by inhomogeneous electric field, spatial fluctuations of electric field can play a major role in the resulting dynamics of electron propagation inside the nanovoid. 


\section{ACKNOWLEDGEMENTS}

\section{This work was supported by the Czech Science Foundation (Project ID 18-04676S).}

\section{REFERENCES}

[1] VELDHUIZEN VAN, E. M. Electrical discharges for environmental purposes : Fundamentals and applications. Huntington: Nova Science, 2000.

[2] ŠUNKA, P. Pulse electrical discharges in water and their applications. Physics of Plasmas. 2001, vol. 8, no. 5, pp. 2587-2594.

[3] JOHNSON, D. L., VANDEVENDER, J. P., MARTIN, T. H. High power density water dielectric switching. IEEE Transactions on Plasma Science. 1980, vol. 8, no. 3, pp. 204-209.

[4] BLUHM, H., FREY, W., GIESE, H., HOPPE, P., SCHULTHEISS, C., STRASSNER, R. Application of pulsed HV discharges to material fragmentation and recycling. IEEE Transactions on Dielectrics and Electrical Insulation. 2000, vol. 7, no. 5, pp. 625-636.

[5] COLEMAN, A. J., CHOI, M. J., SAUNDERS, J. E., LEIGHTON, T. G. Acoustic emission and sonoluminescence due to cavitation at the beam focus of an electrohydraulic shock wave lithotripter. Ultrasound in Medicine \& Biology. 1992, vol. 18, no. 3, pp. 267-281.

[6] LANDAU, L. D., et al. Electrodynamics of continuous media. Oxford, England: Butterworth-Heinemann, 1984.

[7] SHNEIDER, M. N., PEKKER, M. Dielectric fluid in inhomogeneous pulsed electric field. Physical Review E. 2013, vol. 87 , no. 4, p. 043004.

[8] AN, W., BAUMUNG, K., BLUHM, H. Underwater streamer propagation analyzed from detailed measurements of pressure release. Journal of Applied Physics. 2007, vol. 101, no. 5, p. 053302.

[9] BERNAL, M., BORDAGE, M., BROWN, J., DAVIDKOVA, M., DELAGE, E., EL BITAR, Z., ENGER, S., FRANCIS, Z., GUATELLI, S., IVANCHENKO, V., et al. Track structure modeling in liquid water: A review of the Geant4-DNA very low energy extension of the Geant4 Monte Carlo simulation toolkit. Physica Medica. 2015, vol. 31, no. 8, pp. 861-874.

[10] INCERTI, S., IVANCHENKO, A., KARAMITROS, M., MANTERO, A., MORETTO, P., TRAN, H., MASCIALINO, B., CHAMPION, C., IVANCHENKO, V., BERNAL, M., et al. Comparison of geant4 very low energy cross section models with experimental data in water. Medical Physics. 2010, vol. 37, no. 9, pp. 4692-4708.

[11] INCERTI, S., BALDACCHINO, G., BERNAL, M., CAPRA, R., CHAMPION, C., FRANCIS, Z., GUÈYE, P., MANTERO, A., MASCIALINO, B., MORETTO, P., et al. The Geant4-DNA project. International Journal of Modeling, Simulation, and Scientific Computing. 2010, vol. 1, no. 02, pp. 157-178.

[12] INCERTI, S., KYRIAKOU, I., BERNAL, M., BORDAGE, M., FRANCIS, Z., GUATELLI, S., IVANCHENKO, V., KARAMITROS, M., LAMPE, N., LEE, S. B., et al. Geant4-DNA example applications for track structure simulations in liquid water: A report from the geant4-dna project. Medical Physics. 2018, vol. 45, no. 8, pp. e722e739.

[13] YANG, L., ZOU, Y., DA, B., MAO, S., LI, H., ZHAO, Y., DING, Z. A comparative study on monte carlo simulations of electron emission from liquid water. Medical Physics. 2020, vol. 47, no. 2, pp. 759-771. 